\title{
Charmonium in strongly coupled Quark-Gluon Plasma
}

\author{
Clint Young and Edward Shuryak \\ Department of Physics and Astronomy \\ State University of New York, \\ Stony Brook, NY 11794-3800
}

(Dated: November 29, 2018)

\begin{abstract}
The growing consensus that a strongly-coupled quark-gluon plasma (sQGP) has been observed at the SPS and RHIC experiments suggests a different framework for examining heavy quark dynamics. We present both semi-analytical treatment of Fokker-Planck (FP) evolution in pedagogical examples and numerical Langevin simulations of evolving $c \bar{c}$-pairs on top of a hydrodynamically expanding fireball. In this way, we may conclude that the survival probability of bound charmonia states is greater than previously estimated, as the spatial equilibration of pairs proceeds through a "slowly dissolving lump" stage related to the pair interaction.

PACS numbers:
\end{abstract}

\section{INTRODUCTION}

\section{A. Overview}

Charmonium suppression is one of the classical probes used in heavy ion collisions. Since charm quark pairs originate during early hard processes, they go through all stages of the evolution of the system. A small fraction of such pairs $\sim O\left(10^{-2}\right)$ produces bound $\bar{c} c$ states. By comparing the yield of these states in AA collisions to that in pp collisions (where matter is absent) one can observe their survival probability, giving us important information about the properties of the medium.

Many mechanisms of $J / \psi$ suppression in matter were proposed over the years. The first, suggested by one of us in 1978 [1], is (i) a gluonic analog to "photo-effect" $g J / \psi \rightarrow \bar{c} c$. Perturbative calculations of its rate [3] predict a rather large excitation rate of the charmonium ground state. Since charmonia are surrounded by many gluons in QGP, this leads to a conclusion by Kharzeev and Satz 3] that nearly all charmonium states at RHIC should be rapidly destroyed. If so, the observed $\mathrm{J} / \psi$ would come mostly from recombined charm quarks at chemical freezout, as advocated in [4].

However this argument is only valid in weakly-coupled QGP, in which the charm quarks would fly away from each other as soon as enough energy is available. As we will show below, in strongly-coupled QGP (sQGP) propagation of charmed quark is in fact very different. Multiple momentum exchanges with matter will lead to rapid equilibration in momentum space, while motion in position space is slow and diffusive in nature. Persistent attraction between quarks makes the possibility of returning back to the ground state for the $J / \psi$ quite substantial, leading to a substantial survival probablity even after several $\mathrm{fm} / \mathrm{c}$ time in sQGP.

Another idea (ii) proposed by Matsui and Satz [5] focuses on the question of whether charmonium states do or do not survive as a bound state. They argued that because of the deconfinement and the Debye screening, the effective $\bar{c} c$ attraction in QGP is simply too small to hold them together as bound states. Quantum-mechanical calculations by Karsch et al [8] and others have used the free energy, obtained from the lattice, as an effective potential (at $T>T_{c}$ )

$$
F(T, r) \approx-\frac{4 \alpha(s)}{3 r} \exp \left(-M_{D}(T) r\right)+F(T, \infty)
$$

They have argued that as the Debye screening radius $M_{D}^{-1}$ decreases with $T$ and becomes smaller than the root mean square radii of corresponding states $\chi, \psi^{\prime}, J / \psi, \Upsilon^{\prime \prime}, \Upsilon^{\prime}, \Upsilon \ldots$, those states should subsequently melt. Furthermore, it was found that for $J / \psi$ the melting point is nearly exactly $T_{c}$, making it a famous "QGP signal".

These arguments are correct asymptotically at high enough $T$, but the central issue is what happens at (so far experimentally accessible at RHIC) $T=(1-2) T_{c}$. Dedicated lattice studies [9] extracted quarkonia spectral densities using the so called maximal entropy method (MEM) to analyze the temporal (Euclidean time) correlators. Contrary to the above-mentioned predictions, the peaks corresponding to $\eta_{c}, J / \psi$ states remains basically unchanged with $T$ in this region, indicating the dissolution temperature is as high as $T_{\psi} \approx(2.5-3) T_{c}$. Mocsy et al 13] have used the Schrödinger equation for the Green function in order to find an effective potential which would describe best not only the lowest s-wave states, but the whole spectral density. Recently [14 they have argued that a near-threshold enhancement is hard to distinguish from a true bound state: according to these authors, the above mentioned MEM dissolution temperature is perhaps too high.

Another approach to charmonium in heavy-ion collisions, taken by Grandchamp and Rapp [6], does not rely on the perturbative calculation of the excitation crosssection. Charm rescattering is enhanced by formation of bound states in QGP. $J / \psi$ lifetimes were calculated at various temperatures using heavy quark effective theory [7]: the resulting widths are typically a few hundred $\mathrm{MeV}$. If so, the total number of rescatterings of $J / \psi$ in the fireball during its lifetime $(\sim 10 \mathrm{fm} / c)$ is large $(10-30)$. 
This model still has fairly large cross-sections for $J / \psi$ annihilation, so in their so-called two-component model, many of the final charmonia measured are required to originate from statistical coalescence of single charm in the plasma into charmonium states.

There are also other quantum-mechanical studies of the issue since the pioneering paper [8]. Zahed and Shuryak [1] argued that one should not use the free energy $F(T, r)$ as the effective potential, because it corresponds to a static situation in which infinite time is available for a "heat exchange" with the medium. In the dynamical real-time situation they proposed to think in terms of level crossing and Landau-Zener formalism, widely used in various quantum-mechanical applications. In the "fast" limit (opposite to the "adiabatically slow" one) all level crossings should instead be ignored. This corresponds to retaining pure states (described by a wave function rather than density matrix) without the entropy term in $F$, which is nothing else but the internal energy

$$
V(T, r)=F(T, r)-T d F(T, r) / d T=F+T S
$$

instead, as an alternative effective potential. Such potential $V(T, r)$ (extracted from the same lattice data) leads to much more stable bound states, putting charmonium melting temperature to higher $T \sim 3 T_{c}$. A number of authors [12] have used effective potentials in between those two limiting cases. However, as it will be clear from what follows, we think it is not the bound states themselves which are important, but kinetics of transitions between them. In a nutshell, the main issue is how small is the separation in the $\bar{c} c$ pair when the $Q G P$ is over, not in which particular states they have been during this time.

The heavy $Q \bar{Q}$ potential depends not only on the temperature but also on the velocity of the $\bar{c} c$ pair relative to the medium. This effect has been studied e.g. by means of the AdS/CFT correspondence in [15] and it was found that the bound state should not exist above a certain critical velocity. So, if the existence of a bound state is truly a prerequisite for $J / \psi$ survival, one would expect additional suppression at large $p_{t}$. This goes contrary to the well-known formation-time argument [16] and the experimental evidence, indicating the disappearance of the $J / \psi$ suppression at large $p_{t}$. We think this as a good example of how important the real-time dynamics of the $\bar{c} c$ pair in medium is: and indeed we are going to follow it below from its birth to its ultimate fate.

Let us now briefly review the experimental situation. For a long time it was dominated by the SPS experiments NA38/50/60, who have observed both "normal" nuclear absorption and an "anomalous" suppression, maximal in central $\mathrm{Pb}+\mathrm{Pb}$ collisions 19]. Since at RHIC QGP has a longer lifetime and reaches a higher energy density, straightforward extrapolations of the naive $J / \psi$ melting scenarios predicted near-total suppression. And yet, the first RHIC data apparently indicate a survival probability similar to that observed at the SPS.

One possible explanation [20] is that the $J / \psi$ suppression is cancelled by a recombination process from unre- lated (or non-diagonal) $\bar{c} c$ pairs floating in the medium. However this scenario needs quite accurate fine-tuning of two mechanisms. It also would require rapidity and momentum distributions of the $J / \psi$ at RHIC to be completely different from those in a single hard production.

Another logical possibility [21] is that the $J / \psi$ actually does survive both at SPS and RHIC: while the anomalous suppression observed may simply be due to suppression of higher charmonium states, $\psi^{\prime}$ and $\chi$, feeding down about $40 \%$ of $J / \psi$ in pp collisions. These authors however have not attempted to explain why $J / \psi$ survival probablity can be close to one.

This is precisely the goal of the present work, in which we study dynamically how survival of $J / \psi$ happens. We will see that it is enhanced by two famous signatures of sQGP, namely (i) a very small charm diffusion constant and (ii) strong mutual attraction between charmed quarks in the QGP. We found that $J / \psi$ survival through the duration of the QGP phase $\tau \sim 5 \mathrm{fm} / c$ is about a half.

The sequence of events can be schematically described as a four-stage process

$$
\begin{gathered}
\frac{\Longrightarrow}{(\bar{c} \text { c production })} f_{\text {initial }} \frac{\Longrightarrow}{(\text { mom.relaxation })} f_{\text {quasi-equilibrium }}(3) \\
\frac{\Longrightarrow}{(\text { leakage })} f_{\text {final }} \frac{\Longrightarrow}{(\text { projection })} \mathrm{J} / \psi
\end{gathered}
$$

A new element here is a two-time-scale evolution, including rather rapid momentum relaxation to a quasiequilibrium distribution which differs from the equilibrium one at large enough distances.

\section{B. Charmonium potentials at $T>T_{c}$}

The interaction of the $\bar{c} c$ pair will play a significant role in this paper, and thus we briefly review what is known about them. The details can be found in the original lattice results: we will point out only the most important qualitative features.

Perturbatively, at high $T$ one expects a Coulomb-like force, attractive in the color singlet and repulsive in the color octet channel, with the relative strengths 8:(-1) (so that color average will produce zero effect). As shown by one of us many years ago[1], the Coulomb forces are screened by the gluon polarization operator at distances $\sim 1 / g T$.

Quantitative knowledge of the interaction comes from large set of lattice measurements of the free energies associated with a pair of heavy quarks in an equilibrium heat bath. These data include both results by the BielefeldBNL group and in dynamical QCD with $N_{f}=2$ by Aarts et al. 10].

At $T>T_{c}$ one is in a deconfined phase, so at large quark separations one expects effective potentials to go to a finite $V_{\infty}(T)$. Yet when the value of this potential significantly exceeds the temperature, the actual probablity of quark separation is small $\sim \exp \left(-V_{\infty}(T) / T\right)$. As 


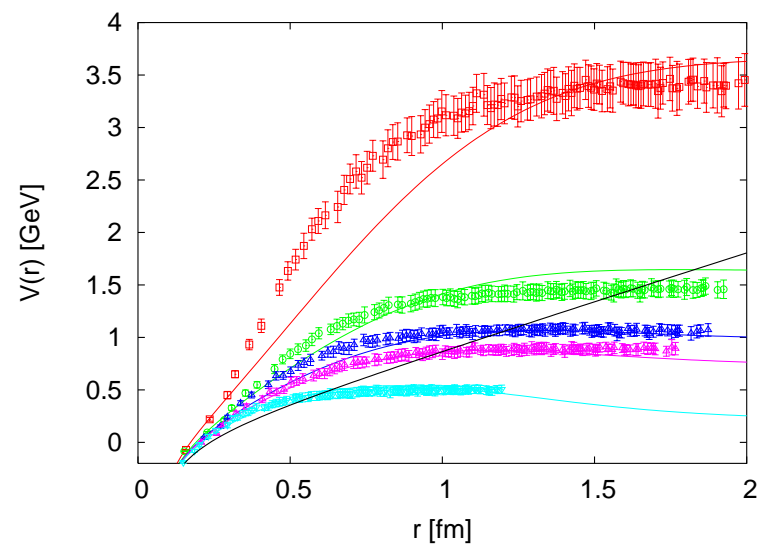

FIG. 1: (Color online.) Parametrization of the potential for lattice data from [24]. From top to bottom: the potential at $T / T_{c}=1.02,1.07,1.13,1.18,1.64$.

we already mentioned in the preceding section, the appropriate potential for dynamical $\bar{c} c$ pair is not yet definitely determined, with suggestions ranging from free energy to potential energy measured on the lattice.

The difference between the two - the entropy associated with $\bar{c} c$ pair - is very large near $T_{c}$, reaching the value $S \sim 20$ at its peak. It means that a huge number of excited states $\sim \exp (S) \sim \exp (20)$ would be excited in adiabatically slow motion of the pair. We think that in realistic motion of $\bar{c} c$ pair much less states are actually excited, and thus, following [11], we will use the potential energy instead of free energy. A cost of this is much larger potential barrier, reducing $\bar{c} c$ dissociation. Indeed, $V_{\infty}(T)$ near $T_{c}$ is large, reaching about $4 \mathrm{GeV}$ at its peak.

For the simulation we need a parametrization of the heavy quark-antiquark potential above deconfinement as a function of temperature and separation. We use the same parametrization as in [13]:

$$
\begin{array}{r}
V(r, T)=-\alpha \frac{e^{-\mu(T) r^{2}}}{r}+\sigma r e^{-\mu(T) r^{2}} \\
+C(T)\left(1-e^{-\mu(T) r^{2}}\right)
\end{array}
$$

and fit to quenched-QCD lattice data in a temperature range of $1.02 T_{c}$ to $1.64 T_{c}$, assuming $\sigma$ is constant in temperature, $\mu$ varies linearly with temperature over this range, and $C(T) \propto\left(T / T_{c}-0.98\right)^{-1}$, so that it peaks sharply at $T_{c}$ as $U_{\text {inf }}$ does. The result shown in Fig $\amalg$ (a) is for

$$
\begin{gathered}
\mu(T)=\left(0.03+0.006 T / T_{c}\right) \mathrm{GeV}^{2} \\
\sigma=0.22 \mathrm{GeV}^{2} \\
C(T)=\frac{0.15 \mathrm{GeV}}{T / T_{c}-0.98}
\end{gathered}
$$

with $\alpha$ set to $\frac{\pi}{12}$.

As you can see from Fig $\mathbb{I B}(a)$, this fit is not perfect. While it is easy to fit a function to a single temperature's data set, it is hard to find an adequate fit across temperatures. This fit however will prove sufficient, especially since it is relatively good for the small separations of interest.

The classical Boltzmann factor $\exp (U(r) / T)$ for a Coulomb potential leads to a non-normalizable distribution. Quantum mechanics prevents this, which can be crudely modeled by an effective potential

$$
V_{e f f}=\hbar^{2} / M r^{2}+V(T, r)
$$

which includes the so called localization energy. Dusling and one of us [17] have determined more accurate effective quantum potentials, following ideas of Kelbg and others and performing path integrals. Perhaps those should be used in future more sophisticated simulations. In the present simulations we simply turn off the force below $0.2 \mathrm{fm}$, approximating the effective potential by a constant.

During the simulation, we allow our pairs to exist as either color singlets or color octets. While in a zerotemperature pp collision, confinement completely suppresses a $\bar{c} c$ pair's probability of existing in a color octet state, in the deconfined phase this possibility must be considered. We initially create a 1:8 ratio of color singlet to color octet pairs, as is expected statistically, and then during each timestep we decide if the pair exists as a color octet by comparing a random number between zero and one to $\frac{1}{Z} \exp \left(-\left(U_{8}-U_{1}\right) / T\right)$, with the color singlet and octet potential energies determined from [38]. In other words, the pairs exist in thermal equilibrium in color space at all times. When a pair exists as a color octet, they do not interact in our simulation as the spatial variation of the octet free energies is quite small.

Naively one would expect this to create a large difference with results where color singlet is assumed for all pairs, however this is not the case. One sees this by noticing that for the temperatures and distances of our interest (distances of separation for pairs likely to go into the $J / \psi$-state), $\frac{U_{8}-U_{1}}{T} \sim 10$, meaning that the octet state is suppressed by orders of magnitude.

Lattice potentials do not follow the simple perturbative relation $V_{1}=-8 V_{8}$ between singlet and octet potentials mentioned above. While the color singlet channel displays a significant attraction, the color octet channel has a potential which is remarkably flat ( $r$-independent). On the basis of this feature of the lattice data we will ignore the force in the octet channel in the simulation.

\section{Charm diffusion constant}

Loosely speaking, the effect we are after in this work can be express as follows: the medium is trying to prevent the outgoing quarks from being separated. It has been conjectured by one of us that charm quark pairs 
should get stopped in QGP [25]. RHIC single electron data suggest that charm quarks, and probably b quarks as well, are indeed equilibrating much more effectively than it was thought before.

The first question one should address is how a charmanticharm pair moves in sQGP, and what is the probablity to find the $\bar{c} c$ in close proximity after time $t$. Charm quarks are subject of three forces: (i) the drag force, trying to reduce the difference between quark momentum distribution and that in (local) equilibrium quark and matter velocities

(ii) stochastic (or Langevin) force from a heat bath, leading to thermal equilibration in momenta and spatial diffusion of the charm quarks

(iii) the $\bar{c} c$ mutual interaction. For pedagogical reasons it is useful to include them subsequently, first for a stationary non-floating matter at fixed temperature (using the Fokker-Planck equation) and then for a realistic nuclear geometry with a hydrodynamically expanding fireball.

But before we do so, let us briefly remind why the case of heavy (charmed) quarks is so special. (More detailed discussion of that can be found in Moore and Teaney [26].) A collision of a heavy quark with quasiparticles of QGP leads to change in its momentum $\Delta p_{H Q} \sim T$, so that the velocity is changed by a small amount

$$
\Delta v_{H Q} \sim T / M_{H Q} \ll 1
$$

Therefore the velocity of the charm quark can only change significantly as a result of multiple collisions, in small steps. Thus the process can be described via appropriate differential equations such as the Fokker-Planck or the Langevin equations. Similarly one can argue that spatial diffusion of a heavy particle can also be described in this way, because the change in position between collisions are small and uncorrelated.

An assumption necessary for Langevin dynamics to hold is that the "kicks" are random (uncorrelated). As explained above, for a single heavy quark it follows from the inequality $M>>T$, which guarantees that the quark relaxation time is long compared to the correlation time in matter. For a $\bar{c} c$ pair we need an additional requirement, that random forces on each quark can be treated as mutually uncorrelated. In order to see how good this approximation is, one should compare the spatial (equal time) correlation length $\xi(T)$ in the medium to the typical distance between quarks for paths which eventually (at the end of the plasma era) will become charmonia. We will provide two different estimates for the former quantity, which view QGP either as a perturbative "gas" or a strongly coupled "liquid".

In a perturbative gas of gluons, the mass is small and in the lowest order momentum distribution is thermal. Thus the maximum of the momentum distribution is at $p \approx 2.7 T$, about $1 \mathrm{GeV}$ at the initial RHIC condition. The corresponding half wavelength (the region where the field keeps at least its sign)

$$
\lambda / 2=\pi / p \approx .6 \mathrm{fm} \sim \xi(T=.4 \mathrm{GeV})
$$

In the liquid regime quasiparticles do not successfully model the degrees of freedom. However we do have phenomenological information about spatial correlations from hydrodynamics, which propose the so called "sound absorption length" as a measure above which different matter "cells" decorrelate. It is

$$
\Gamma_{s}=\frac{4 \eta}{3 s T}
$$

with $\eta / s$, the dimensionless ratio of viscosity to entropy density. Empirically, RHIC data are well described if it is of the order of $1 / 3 \pi$ (the AdS/CFT strong-coupling limit), which suggests a spatial correlation length one order of magnitude smaller $\xi(T=.4 \mathrm{GeV}) \sim 0.05 \mathrm{fm}$.

Since the distance between $\bar{c}$ and $c$ which eventually become $J / \psi$ is about $.5 \mathrm{fm}$, and since there are good reasons to believe the latter "liquid" estimate of $\xi$ is closer to reality, we conclude that the main Langevin assumption - the independence of random forces for $\bar{c}$ and $c$ - is well justified. Furthermore, one may think that the same assumption would even hold for $\bar{b}$ and $b$, although with worse accuracy.

Since this small parameter in $\mathrm{Eq} 9$ is central to what follows, let us remind for orientation of the reader that at RHIC we speak about the ratio for charmed quark $T / M_{c}=(1 / 6-1 / 5)$, or $T / M_{b}=(1 / 20-1 / 15)$ for $b$ quark.

Although RHIC experiments with charm quarks include direct reconstruction of charmed mesons $D, D^{*}$ by the STAR collaboration, so far the existing vertex detectors are not sufficient for doing it effectively (upgrades are coming). Therefore the most relevant data on charm is based on observation of single electrons from heavy quark weak semileptonic decays. Apart of electromagnetic backgrounds, we do not really know whether electrons come from $c$ or $b$ decays: it is believed (but not yet proven) that the boundary between two regimes is at $p_{t} \approx 4 \mathrm{GeV}$. Two experimentally observable quantities are (i) the charm suppression relative to the parton model (no matter) $R_{A A}^{e}$ and (ii) the azimuthal asymmetry of the electrons relative to impact parameter $v_{2}^{e}=\langle\cos (2 \phi)\rangle$.

Several theoretical groups have analyzed these data, in particular Moore and Teaney [26] provided information about the diffusion constant of a charm quark $D_{c}$ by Langevin simulations. A conclusion following from this work is that both $R_{A A}^{e}$ and $v_{2}^{e}$ observed at RHIC can be described by one value for the charm diffusion constant in the range

$$
D_{c}(2 \pi T)=1.5-3 .
$$

This can be compared with the perturbative (collisional) result at small $\alpha_{s}$

$$
D_{c}^{p Q C D}(2 \pi T)=1.5 / \alpha_{s}^{2} .
$$

Assuming that the perturbative domain starts $^{1}$ some-

\footnotetext{
${ }^{1}$ Recall that at $4 / 3 \alpha_{s}=1 / 2$ two scalar quarks should fall towards
} 
where at $\alpha_{s}<1 / 3$ one concludes that the empirical value (12) is an order of magnitude smaller than the perturbative value.

There are two studies of the diffusion constant at strong coupling. One comes from AdS/CFT correspondence [27] (and thus the results are for $\mathcal{N}=4$ supersymmetric Yang-Mills theory): the final expression found by Casalderrey-Solana and Teaney [28] is

$$
D_{H Q}=\frac{2}{\pi T \sqrt{g^{2} N_{c}}}
$$

It is nicely consistent (via the Einstein relation) with the calculated drag force

$$
\frac{d P}{d t}=-\frac{\pi T^{2} \sqrt{g^{2} N_{c}} v}{2 \sqrt{1-v^{2}}}
$$

by Herzog et al [29]. One assumption of this calculation is that the 't Hooft coupling is large $g^{2} N_{c} \gg 1$, which means the diffusion constant is parametrically small, much less than momentum diffusion in the same theory $D_{p}=\eta /(\epsilon+p) \sim 1 / 4 \pi T$. This result is also valid only for quarks heavy enough

$$
M_{H Q}>M_{e f f} \sim \sqrt{g^{2} N_{c}} T
$$

which only marginally holds for charm quarks.

Let us see what these numbers mean for RHIC (assuming that they are valid for QCD). The 'tHooft coupling $g^{2} N_{c}=\alpha_{s} 4 \pi N_{c} \approx 20-40$ is indeed large, while $M_{\text {eff }} \approx 1-2 \mathrm{GeV}$ is not really small as compared to the charm quark mass: thus the derivation is only marginally true. Yet we proceed and get

$$
D_{c}(2 \pi T)=4 / \sqrt{g^{2} N_{c}} \sim 0.5-1,
$$

which is in right ballpark of phenomenological numbers.

Another approach to transport properties of a strongly coupled plasmas is classical molecular dynamics. A classical non-Abelian model for sQGP was suggested by Gelman et al [30], and recently Liao and Shuryak [31] have added magnetically charged quasiparticles. Those calculations also find that the diffusion constant $D$ rapidly decreases as a function of the dimensionless coupling constant $\Gamma$ as a power

$$
D \sim\left(\frac{1}{\Gamma}\right)^{0.6-0.8}
$$

in a liquid domain $\Gamma=1-100$. Qualitatively it is similar to the $1 / \sqrt{g^{2} N_{c}}$ of the AdS/CFT result (17). Unfortunately, at this moment there is no deep understanding of the underlying mechanism of both strong coupling calculations.

But this is well beyond the aims of the present work: in what follows we will use $D_{c}(2 \pi T)=1.5,3$ as a range for our best current guess.

each other, according to the Klein-Gordon equation: so this is clearly not a perturbative region.

\section{CHARMONIUM IN SQGP: FOKKER-PLANCK FORMALISM}

Before we described realistic Langevin simulations of RHIC collisions it is useful to see first the basic features analytically. In this section we describe how (1) the high drag coefficient $\eta_{c}$ causes rapid thermalization of the initially hard momentum distribution from PYTHIA, and (2) the small diffusion coefficient $D_{c}$, inversely proportional to $\eta_{c}$, combined with (3) the use of the static $Q \bar{Q}$ internal energy instead of the free energy for the pair interaction leads to a slow dissolution of a large peak in the distribution in position space, causing less suppression than expected by more naive models.

To see the first feature, rapid thermalization in momentum space, consider a $\bar{c} c$ pair with the quarks emerging back-to-back from a hard process with momentum $\mathbf{p}>>T$, therefore having relative momentum $\Delta \mathbf{p}=2 \mathbf{p}$. For $\mathbf{p}>>T$, as is the case initially for charm quarks created at the RHIC, we may neglect the random kicks that the quarks experience from the medium and consider only the drag: $\frac{d(\Delta \mathbf{p})}{d \tau}=-\eta_{c} \mathbf{p}$, where $\eta_{c}=\frac{T}{M_{c} D_{c}}$. This leads to a very simple formula for the relative momentum of a pair at early times:

$$
\Delta \mathbf{p}(t)=\exp \left(-\eta_{c} \tau\right) \Delta \mathbf{p}(0) .
$$

Using the AdS/CFT value for the diffusion coefficient, this leads to a drag coefficient $\eta_{c} \approx .6 \mathrm{GeV}$.

Let us now examine the distribution of $\bar{c} c$ pairs created with PYTHIA pp-event generation at energies of $200 \mathrm{GeV}$ (see more on this in Section III). The initial transverse momentum distribution ${ }^{2}$ is broad compared to the thermal distribution, and therefore we may apply Eq. 19 for early times. We parametrize the initial relative momentum distribution as in Section IIIB and replace $\Delta \mathbf{p}$ with $\Delta \mathbf{p} \exp \left(\eta_{c} \tau\right)$, which is the formula for a pair's initial relative momentum in terms of its relative momentum at proper time $\tau$. Next, we compute the overlap of this distribution at early times with the $J / \psi$-state's Wigner quasi-probability distribution to determine the probability of a random $\bar{c} c$ taken from this ensemble to be measured as a $J / \psi$ particle, an approach detailed in Appendix B.

Fig. II shows this probability as a function of time for $\eta_{D}=0.88$. The initial value of the projection is $0.8 \%$, on the same order of magnitude as the experimental value of $1 \%$ for $\frac{\sigma_{J / \psi}}{\sigma_{c \bar{c}}}$ obtained from [36], [37]. The projection increases as the PYTHIA-with-drag distribution narrows but then drops when the width shrinks more than the $J / \psi$ 's width in momentum space and as the

\footnotetext{
2 The initial rapidity distribution is wide also, but since longitudinally Bjorken-like hydrodynamics starts immediately, a charm quark finds itself with comoving matter with the same rapidity, and thus has little longitudinal drag. Transverse flow is slow to be developed, thus there is transverse drag.
} 


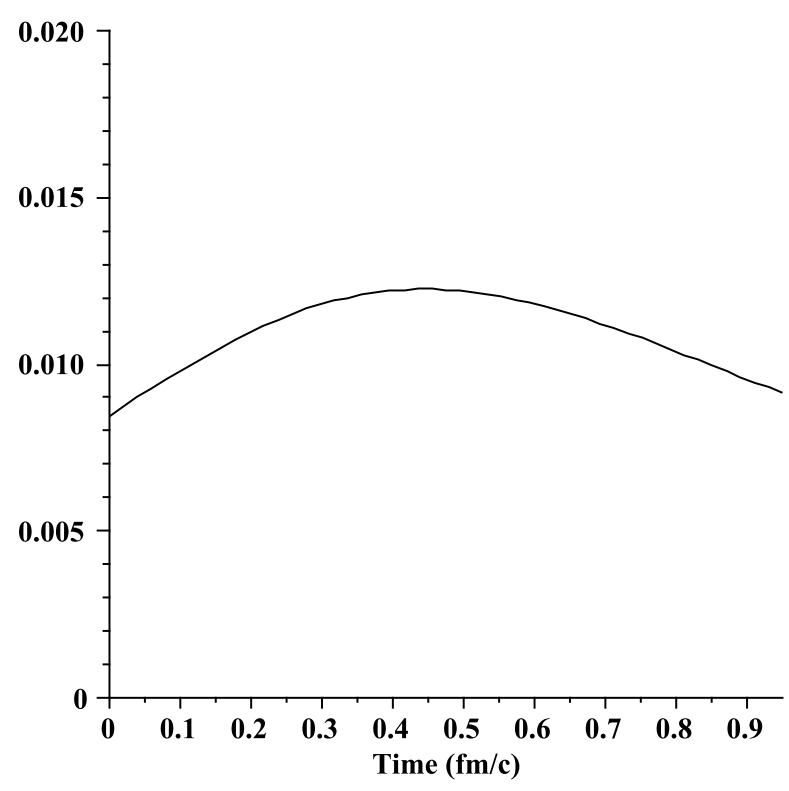

FIG. 2: Probability of a $\bar{c} c$ pair going into the $J / \psi$-state vs. time, for very early time.

quark pair's separation increases. Once the time reaches about $1 \mathrm{fm} / \mathrm{c}$, the probability for a pair to go into a $J / \psi$-state is about where we started, and we quit looking at this approach after this much time because the mean transverse momentum for a quark is the thermal average.

After this first $1 \mathrm{fm} / \mathrm{c}$ of the QGP phase, the $\bar{c} c$ distribution has thermalized in momentum space and the evolution in position space (diffusion) needs to be examined. The root mean square distance for diffusive motion is given by the standard expression

$$
\left\langle x^{2}\right\rangle=6 D_{c} \tau
$$

where $\tau$ is the proper time and the interaction between the quarks has been neglected. The "correlation volume" in which one finds a quark after time $\tau$ is

$$
V_{\text {corr }}=\frac{4 \pi}{3}\left(6 D_{c} \tau\right)^{3 / 2}
$$

and one may estimate for the probability of the $\bar{c} c$ pair to be measured in the $J / \psi$-state as

$$
P(\tau) \sim R_{J / \psi}^{3} /\left(6 D_{c} \tau\right)^{3 / 2}
$$

So neglecting the pair's interaction leads to a small probability that $J / \psi$-states will survive by the hadronization time at the RHIC $(\tau \sim 10 \mathrm{fm} / c)$, even for small values of the diffusion coefficient.

To get an idea for how this simple result is changed by the inclusion of an interaction between the constituent quarks in a given $\bar{c} c$-pair, let us examine the FokkerPlanck equation for the $\bar{c} c$ distribution in relative position:

$$
\frac{\partial P}{\partial t}=D \frac{\partial}{\partial \mathbf{r}} f_{0} \frac{\partial}{\partial \mathbf{r}}\left(P / f_{0}\right)
$$

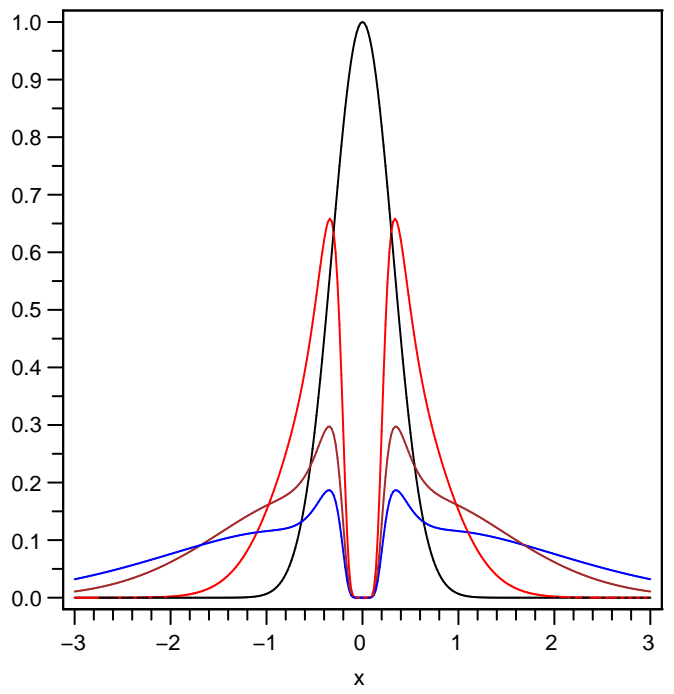

FIG. 3: (Color online.) Numerical solution of the one-dimensional Fokker-Planck equation for an interacting $\bar{c} c$ pair. The relaxation of the initial narrow Gaussian distribution is shown by curves (black, red,brown,green, blue, or top to bottom at $\mathrm{r}=0$ ) corresponding to times $t=0,1,5,10 \mathrm{fm}$, respectively.

where $f_{0}(r) \propto \exp \left(-V_{\text {eff }}(r) / T\right)$ is the equilibrium distribution in the magnitude of relative position $r$. By substituting the potential shown above at $T=1.25 \mathrm{Tc}$ and $D_{c} \times(2 \pi T)=1$ into the Fokker-Planck equation (for demonstration in a single spatial dimension only) we solve it numerically and find how the relaxation process proceeds. A sample of such calculations is shown in Fig. II] It displays two important features of the relaxation process:

(i) during a quite short time $T, 1 \mathrm{fm}$ the initial distribution (peaked at zero distance) relaxes locally to the nearequilibrium distribution with two peaks, corresponding to optimal distances of the equilibrium distribution $f_{0}$, where the effective potential is most attractive;

(ii) the second stage displays a slow "leakage", during which the maximum is decreasing while the tail of the distribution at large distances grows. It is slow because the right-hand side of the Fokker-Planck equation is close to zero, as the distribution is nearly $f_{0}$. The interaction drastically changes the evolution of the $\bar{c} c$ distribution in position space, and this will be demonstrated again in the full numerical simulation of the next section. 


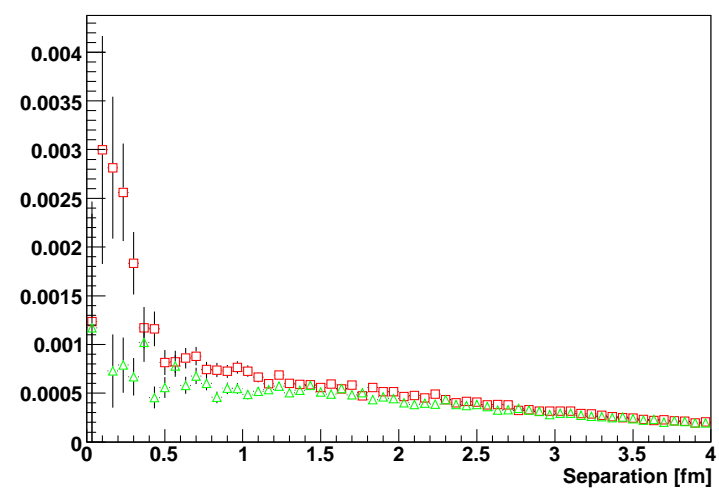

FIG. 4: (Color online.) Distribution over quark pair separation at fixed $T=1.5 T_{c}$ after $9 \mathrm{fm} / \mathrm{c}$, with (red squares) and without (green triangles) the $\bar{c} c$ potential.

\section{LANGEVIN EVOLUTION OF THE $\bar{c} c$ PAIRS}

\section{A. Langevin evolution in static medium: quasiequilibrium}

Before we turn to expanding fireball, we first study the evolution of $\bar{c} c$ pairs at fixed $T=1.5 T_{c}$ and in the absence of hydrodynamical flows. The first thing we would like to demonstrate is the strong influence of the heavy quark interaction. The resulting distribution over interquark separation at time $10 \mathrm{fm} / c$ are shown in Fig. III A with the interaction (red squares) and without (blue triangles). The value for a given pair's separation $r$ is weighted by $\frac{1}{r^{2}}$ so that the spatial phase space of the distribution is divided out. With the interaction on, we find the same behavior of a "slowly dissolving lump" as that seen in the solution of the Fokker-Planck equation when the interaction is "turned on".

Further study of this has shown convergence of its shape to a particular one, which persist for a long time and which we would call "quasiequilibrium" ${ }^{3}$. While the true equilibrium of course corresponds to complete dissolution of a single $\bar{c} c$ pair, it turns out that leakage to large distances affects the distributions of separation and energy in normalization only. In Fig. IIIA is the energy distribution for the ensemble of pairs at $\tau=9 \mathrm{fm} / c$ after evolving under Langevin dynamics at a fixed temperature $1.05 T_{c}$, and it is shown to be the same distribution, up to normalization and statistical uncertainty, as the distribution reached by the pairs in a full heavy-ion simulation of the most central collisions.

\footnotetext{
3 This situation should not be confused with stationary nonequilibrium solutions of the Fokker-Planck equation, in which there is a constant flow through the distribution because of matching source and sink.
}

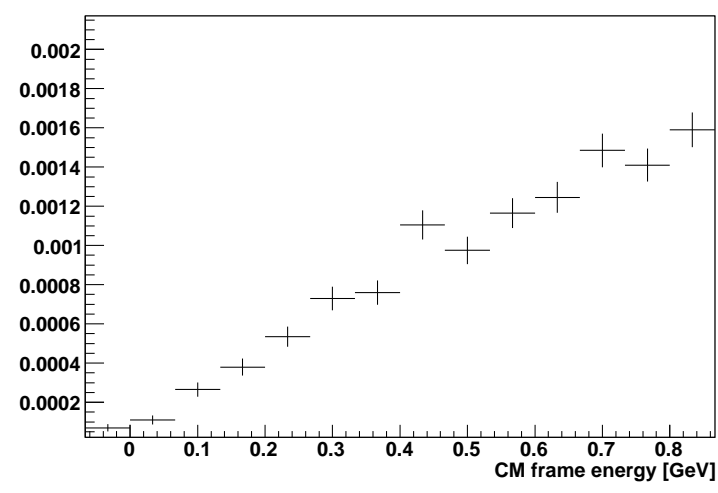

FIG. 5: Energy distribution of the $c \bar{c}$ pairs (in the center of mass frame of the pair) after Langevin evolution at a fixed temperature $T=1.05 T_{c}$ for a time $t=9 \mathrm{fm} / c$ long enough for quasiequilibrium to be reached.

We show the energy distribution in this region because it is related to a very important issue of the charmonium production, namely production of $\psi^{\prime}, \chi$ states and subsequent feeddown into the $J / \psi$. When a quasiequilibrium distribution is reached, the production ratios of charmonium states are stabilized at thermal (statistical) model values, in spite of the fact that the overall normalization continues to shrink due to leakage into infinitely large phase space at large distances.

(The energy distribution itself contains a Boltzmann factor but also the density of states. A model case of purely Coulomb interaction allows one to calculate it in a simple way: as shown in Appendix $\mathrm{A}$ we found that in this case the absolute shape of the quasiequilibrium distribution is reproduced as well.)

The existence of quasiequilibrium is in good correspondence to observations. It was noticed already a decade ago [42] that the SPS data on centrality dependence of $N_{\psi^{\prime}} / N_{J / \psi}$ ratio approached the equilibrium value (for chemical freezout)

$$
\frac{N_{\psi^{\prime}}}{N_{J / \psi}}=\exp \left(-\Delta M / T_{c h}\right)
$$

with the chemical freezout at $T_{c h}=170 \mathrm{MeV}$, as is observed for ratios of other hadronic species.

One possible explanation of it can be charmonium recombination (from independent charm quarks) at chemical freezout, advocated by [4] and others. However our findings show that the same ratio naturally appears even for a single $\bar{c} c$ pair dissolving in a thermal medium, in a "quasiequilibrium" occurring at the leakage stage. Especially at SPS, when statistical recombination requires a charm density which is too large, this is an attractive possibility. 


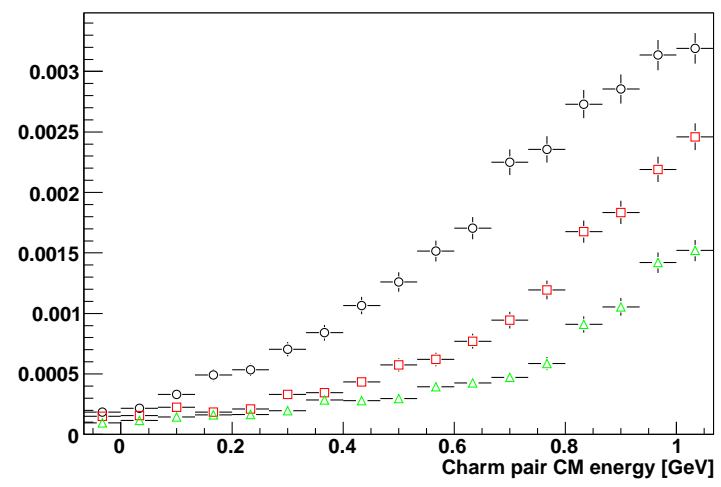

FIG. 6: (Color online.) Evolving energy distribution for an ensemble of $\bar{c} c$ pairs at time moments $t=2,3,10 \mathrm{fm} / \mathrm{c}$ (circles,squares and triangles, respectively).

\section{B. Production of the initial $\bar{c} c$ pairs}

We start with $\bar{c} c$ events produced with PYTHIA, a particle physics event generator [33]. PYTHIA yields $\bar{c} c$ pairs through a set of perturbative QCD events: through Monte-Carlo it will select initial partons from the parton distribution function of one's choosing and proceed through leading-order QCD differential cross sections to a random final state. The $p_{t}$ and rapidity distributions of charm produced in pp collisions is believed to be rather adequately represented.

By using PYTHIA we do not however imply that it solved many open issues related with charm production, such as color octet versus singlet state. It also leaves open the very important issue of feeddown from charmonium excited states (see below). One more open question needed to start our simulations - is how to sample the distribution in position space. Indeed, each pQCD event generated in PYTHIA is a momentum eigenstate without any width, so by Heisenberg's uncertainty relation they are spatially delocalized, which is unrealistic.

We proceed assuming the form for the initial phasespace distribution to be

$$
P_{\text {init }}(\mathbf{r}, \mathbf{p}) \propto P_{\mathrm{PYTH}}(\mathbf{p}) \exp \left(-\mathbf{r}^{2} / 2 \sigma^{2}\right)
$$

By setting $\sigma=0.3 \mathrm{fm}$ one can tune energy distribution to give a reasonable probability for the formation of $J / \psi$ state in pp events.

However this does not yield correct formation probabilities of $\chi, \psi^{\prime}$. It is hardly surprising, since for example the $\psi^{\prime}$ wavefunction has a sign change at certain distances, and the exact production amplitude is required for a projection, an order-of-magnitude size estimate is not good enough. Since feeddown from those states contributes about $40 \%$ of the observed $J / \psi$, we simply refrain from any quantitative statements about pp (and very peripheral AA) collisions, focusing only on distributions after some amount of time spent in QGP.

\section{Langevin motion of $\bar{c} c$ pair in an expanding fireball}

Finally, we model the motion of a charm quark pair in an evolving fireball. We use the same framework and programs used in [26] to examine motion of a single charm quark for propagation of an interacting pair.

We start with large number of $\bar{c} c$ produced with PYTHIA pQCD event generation, and randomly place them in position space throughout the fireball, using a Monte-Carlo Glauber calculation.

Then, the pairs are evolved in time according to the Langevin equations:

$$
\frac{d \mathbf{p}}{d t}=-\eta \mathbf{p}+\xi-\nabla U
$$

$$
\frac{d \mathbf{r}}{d t}=\frac{\mathbf{p}}{m_{c}}
$$

where $\xi$ corresponds to a random force and $\eta$ the drag coefficient. The condition that the Langevin equations evolve a distribution towards thermal equilibrium gives the relation $\left\langle\xi_{i}(t) \xi_{j}\left(t^{\prime}\right)\right\rangle=2 M T \eta \delta_{i j} \delta\left(t-t^{\prime}\right)$. We proceed here with our diffusion constant set by the results of [26] to be $\eta_{D}=\frac{2 \pi T^{2}}{1.5 M}$. and as discussed earlier with our potential as $V$ instead of $F$.

Now we examine the evolution of the quark pairs as discussed before, examining pairs at mid-rapidity in a boost-invariant, 2-dimensional ultra-relativistic gas simulation, the same hydrodynamical simulation used in [26]. We stop the Langevin-with-interaction evolution when $T<T_{c}$. The distribution over energy at different moments of the time is shown in Fig IIB

We will discuss our results subsequently, at two different levels of sophistication. First we will show results with only the total number of bound states monitored, and then we will show results where the different charmonia states and feeddown to $J / \psi$ are considered and compare these results with PHENIX data.

How we determine whether or not to call a $\bar{c} c$ pair in our simulation bound, and what particular charmonium state the pair exists as if it is bound, is discussed in Appendix B. Fig. IIIC shows the number of "bound" $\bar{c} c$ pairs as a fraction of their total number, monitored throughout the course of the simulation as a function of time. Note that realistic hydrodynamical/Langevin simulation agrees qualitatively with the analytic results of Section III During the first $\mathrm{fm} / \mathrm{c}$ one finds some boosts in the probability for a $\bar{c} c$ pair to be bound, due to rapid thermalization in momentum space. Later the probablity falls due to the slow diffusion in position space. This figure emphasizes our main qualitative finding, the survival probability of $J / \psi$ on the order of a half. 


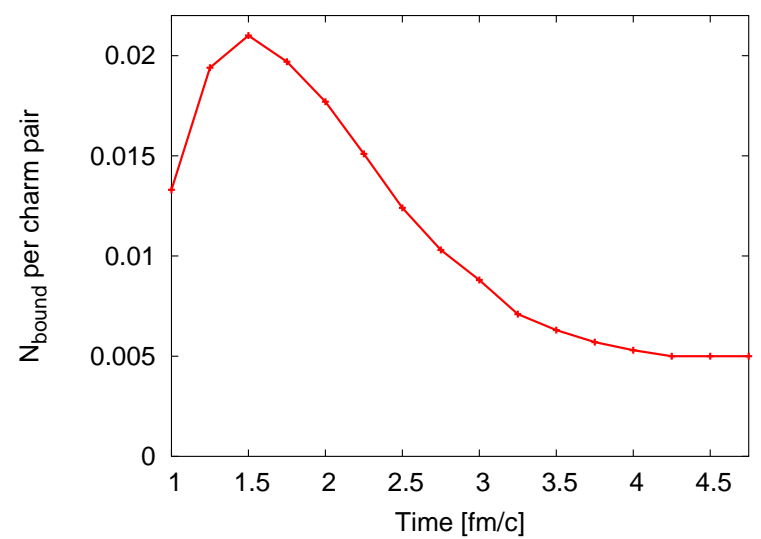

FIG. 7: (Color online.) Probability of $\bar{c} c$ pairs to be bound at $\mathrm{RHIC} \mathrm{Au}+\mathrm{Au}, \sqrt{s}=200 \mathrm{GeV}$, mid-rapidity.

\section{Shadowing and "normal" absorption}

Experimental data include not only the "QGP suppression" we study in this work, but also (i) the initialstate effects (modified parton distributions in "cold nuclear matter") plus (ii) the so called "normal nuclear absorption". The way we have chosen to display PHENIX data [41] is as follows: before we compare those with our results we "factor out" the cold nuclear matter effects, by defining (for any given rapidity $y$ ) the following ratio of $\mathrm{Au}+\mathrm{Au}$ and $\mathrm{d}+\mathrm{Au}$ data

$$
R_{A A}^{\text {anomalous }}(y)=\frac{R_{A A}^{P H E N I X}(y)}{R_{p A}(y) R_{p A}(-y)}
$$

to be called the "anomalous suppression". In principle those include only data: but unfortunately the large dAu sample taken in 2008 is not yet analyzed (at the time of this writing), while the 2003 set has error bars which are too large. This forces us to use a model at this point, following Kharzeev et al 39] with $R_{p A}=\exp \left(-\sigma_{a b s}\langle L\rangle n_{0}\right)$, where $\langle L\rangle$ is the mean path length of the $J / \psi$ through nuclear matter, $n_{0}$ is the nuclear density, and $\sigma_{\text {abs }}$ is the nuclear absorption cross-section (parametrized from [39] to be $0.1 \mathrm{fm}^{2}$ for rapidity $\left.y=0\right)$. Finally, for rapidity $y=0$, we rewrite this as $\left(R_{p A}(y=0)\right)^{2}=\exp \left(-\sigma_{a b s}\left\langle N_{\text {part }}\right\rangle\right.$, where $\left\langle N_{\text {part }}\right\rangle$ is the density per unit area of participants in the collision plane. We further used Glauber model calculations [40] to determine $\left\langle N_{\text {part }}\right\rangle$ for a given $N_{\text {part }}$ at PHENIX. We divide each $\mathrm{Au}+\mathrm{Au}$ data point from PHENIX by this quantity and call it $R_{A A}^{\text {anomalous }}(y=0)$ plotted as points in Figs IID and 10, to be compared with our simulation.

\section{E. $\psi^{\prime}$ production and feeddown}

Next we calculate the ratio $N_{\psi^{\prime}} / N_{J / \psi}$ in our simulations, for different centralities. There are well known NA38/50/60 measurements of this ratio at the SPS, but

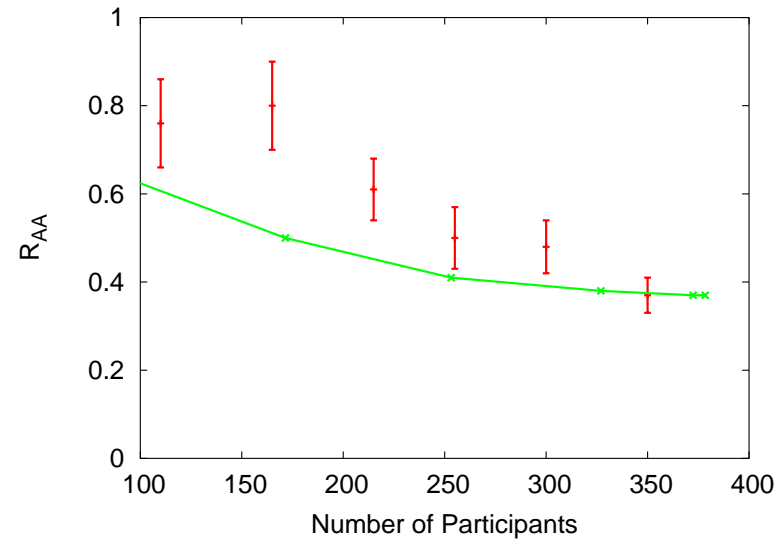

FIG. 8: (color online) Points show the magnitude of the anomalous suppression at mid-rapidity $R_{A A}^{\text {anomalous }}(y=0)$ versus the centrality (the number of participants), using $\mathrm{Au}+\mathrm{Au}$ PHENIX data. The curve is the probability to be bound (determined by the energy projection) at the end of the QGP era, when $T=T_{c}$.

at RHIC it has been measured so far only in pp collisions by the PHENIX detector [44] to be $0.14 \pm 0.04$, which makes the ratio of direct $\psi^{\prime}$ to $J / \psi$ particles 0.24 as in [43]. Hopefully higher luminosity at RHIC will make possible a future measurement of this ratio in $\mathrm{Au}+\mathrm{Au}$ collisions of various centralities.

We calculate $N_{\psi^{\prime}} / N_{J / \psi}$ as follows: (i) first we run our simulation and determine the distributions $f(E)$ over the $\bar{c} c$ pair energy $E=E_{C M}-2 M_{c}$ (in the pair center of mass frame); (ii) then we compare those to quasiequilibrium ones from simulations at fixed temperature (slightly above $\left.T_{c}\right) f_{0}(E)$. Since both are done for the same interaction, in the ratio $f(E) / f_{0}(E)$ the density of states drops out. This ratio tells us how different the actual distribution is from that in quasiequilibrium. (iii) Then we form the double ratios, at two relative energies corresponding to $\psi^{\prime}$ and $J / \psi$ masses (minus $2 m_{\text {charm }}$ )

$$
R_{\psi^{\prime} / J \psi}=\frac{f(.8 \mathrm{GeV})}{f_{0}(.8 \mathrm{GeV})} / \frac{f(.3 \mathrm{GeV})}{f_{0}(.3 \mathrm{GeV})}
$$

This now includes nonequilibrium effects for both of them. Finally (iv) we switch from continuum classical distributions to quantum one, assuming that in quasiequilbrium the relation (24) holds. If so, the particle ratio is a combination of nonequilibrium and equilibrium factors

$$
\frac{N_{\psi^{\prime}}}{N_{J / \psi}}=R_{\psi^{\prime} / \psi} \exp (-\Delta m / T)
$$

The double ratio ( or $\exp (\Delta M / T) N_{\psi^{\prime}} / N_{J / \psi}$ ) is plotted vs centrality in Fig. 9, As one can see, it goes to unity for the most central collisions: so quasiequilibrium is actually reached in this case. For mid-central bins the $\psi^{\prime}$ production is about twice larger because of insufficient time. This is to be compared to the experimental pp 


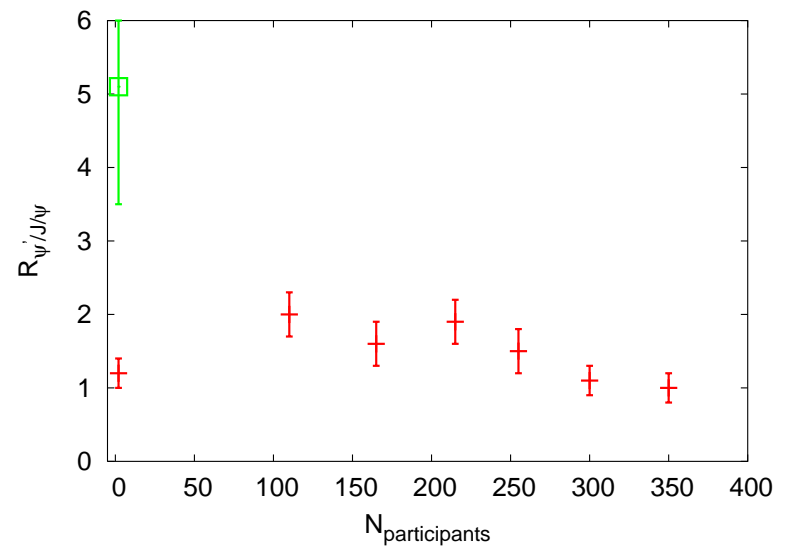

FIG. 9: (Color online.) The double ratio $R_{\psi^{\prime} / \psi}$ defined in (29) versus centrality (number of participants). One point (green box) at $N_{\text {part }}=2$ corresponds to experimental data for $\psi^{\prime}$ and the direct $J / \psi$, for pp collisions.

value for the ratio, which is about 5. (We remind the reader that PYTHIA plus our classical projection method does not work for pp collisions.)

Finally, we use this result to estimate the effect of feeddown from higher states. To do this, we write the final number of $J / \psi$ particles observed as the number of directly produced $J / \psi$ particles plus the number of $J / \psi$ particles produced from feeddown from higher charmonium states:

$$
N_{J / \psi}^{f i n a l}=N_{J / \psi}^{\text {direct }}\left[1+R_{\psi^{\prime} / \psi} \sum_{i}\left(\frac{g_{i}}{3}\right) \exp \left(-\frac{\Delta M_{i}}{T}\right) B_{i}\right](31)
$$

where $i$ is summed over the $\chi_{1}, \chi_{2}$, and $\psi^{\prime}$ particles which contribute significantly to feeddown, $B_{i}$ represents their branching ratio into $J / \psi+\ldots$, and $g_{i}$ is the degeneracy of the state (divided by 3 , the degeneracy of the $J / \psi$ ), $\Delta m_{i}$ is the mass difference between the i-th state and the $J / \psi$. The $R_{\psi^{\prime} / \psi}$ is the non-equilibrium factor discussed above: it is factored outside the sum because it is very similar for all these states.

Now we are ready to discuss centrality dependence of the $J / \psi$ production including the feeddown. We define for each centrality direct $N_{J / \psi}^{\text {direct }}(b)$ as the total number of $c \bar{c}$ pairs in our ensemble with energy (in its rest frame) less than $E<2 M_{\text {charm }}+0.33 \mathrm{GeV}$. The feedown gets its dependence on centrality from $R_{\psi^{\prime} / \psi}(b)$ determined from simulation.

The absolute normalization of the results deserves special discussion. We predict the absolute probability of $J / \psi$ production, both direct and with feeddown, normalized per $\bar{c} c$ pair produced in the same collisions (e.g. centrality bin). Unfortunately, the total cross section of charm production is not yet measured with sufficient accuracy to normalize results this way.

The usual way to present these results is in the form of the so called $R_{A A}$ ratio, relating production in $\mathrm{Au}+\mathrm{Au}$

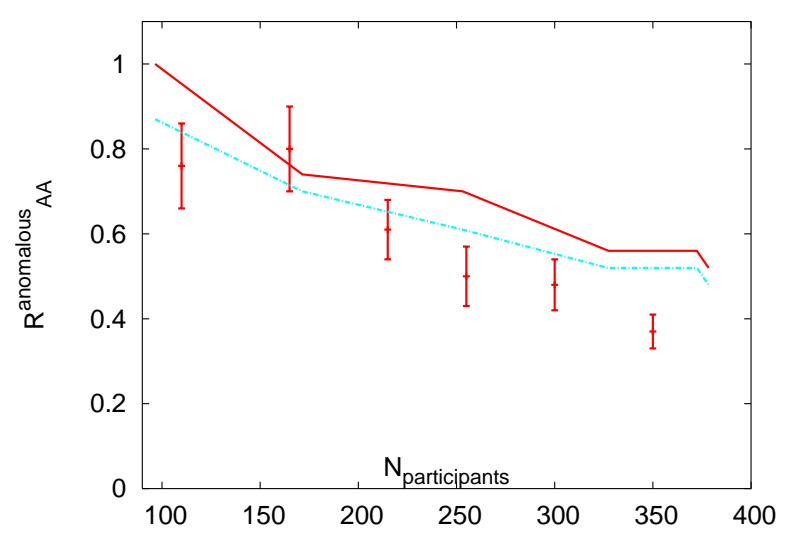

FIG. 10: (Color online.) The points are PHENIX data for $R_{A A}^{\text {anomalous }}(y=0)$, the same as used in Fig IIID. Two curves are our model, with (solid, upper) and without (dashed, lower) feeddown.

at given centrality to that in $\mathrm{pp}$, times the theoretical (Glauber) predictions for the number of hard collisions. In other words, the parton model for $\bar{c} c$ is used. Unfortunately, the experimental data about feeddown in pp are still uncertain enough to produce sufficiently large scale ambiguity. We cannot obtain this from our theory as well because (as explained above) our classical projections do not work adequately for pp (and very peripheral) collisions. Thus we see at the moment no sufficiently accurate way of absolute comparison with the data. Because of that, we simply normalize our results assuming that there is no QGP suppression ( meaning $\left.R_{A A}^{\text {anomalous }}\left(N_{\text {part }}<100\right)=1\right)$. The results normalized like this are shown in Fig. 10, we conclude that although feeddown is not large, taking it into account helps bring the shape of the anomalous suppression closer to observations.

\section{F. Including the "mixed phase"}

In our work so far, we have only examined the evolution of the $c \bar{c}$ pairs during the QGP phase, stopping the evolution wherever the fluid's temperature reached $T_{c}$. However, in order to understand the evolution of charmonia to their hadronization we need to model the dynamics of the charm quarks also through the "mixed phase", also known as the near- $T_{c}$ region.

In various hydrodynamic models which describe heavy ion collisions, the region of roughly $T=0.9-1.1 T_{c}$ is treated as a separate "mixed phase" distinct from QGP and hadronic phases. Indeed, it has a very different equation of state: while the temperature and pressure remain nearly constant, the energy and entropy densities jump 
by a large factor ${ }^{4}$.

What is very important for the present paper is that the near- $T_{c}$ region occupies a significant fraction of the fireball evolution, in spite of being a very narrow interval in terms of $T$. Indeed, one should rather focus not on $T$ but on the entropy density $s$, which shows a simple monotonous decrease with time $s \sim 1 / \tau$ for all three phases.

For a quantitative description of the mixed phase we used hydrodynamical calculations, known to describe radial and elliptic flow well, such as the work by Shuryak and Teaney [45]. It follows from their solution that the "mixed phase" persists for about $5 \mathrm{fm} / c$ after the deconfined phase, which is comparable to the lifetime of the deconfined phase at the very center of the fireball. Thus it is by no means a small effect, and should be included in any realistic treatment of a heavy-ion collision.

The flow during this time was found to be well approximated by a Hubble-like expansion with radial velocity $v=H r$ and time-independent $H \approx 0.075 \mathrm{fm}^{-1}$ for central collisions. For a collision with a nonzero impact parameter (below we will consider $b=6.5 \mathrm{fm}$ ), the anisotropy of this expansion can be parameterized similarly:

$$
v_{i}=H_{i} x_{i}
$$

with $i=1,2$ and $H_{x}=0.078 \mathrm{fm}^{-1}, H_{y}=0.058 \mathrm{fm}^{-1}$ : thus anisotropy is only about $80 \%$ by this late stage. It is fair to say that we have a fairly reasonable understanding of how the medium flows for these later stages: thus in our simulations we have used those parameterizations instead of numerical solutions to hydrodynamics, which were necessary for the QGP phase.

Let us start with two extreme scenarios for the dynamics of the charm quarks during this phase of the collision: 1.) the charm quarks are completely "stopped" in the medium, so that they experience the same Hubble-like flow as matter;

2.) $\bar{c} c$ pairs do not interact at all with the medium near$T_{c}$, moving ballistically with constant velocity for the corresponding time in the collision.

If the first scenario were true, the effect of Hubble flow would be to increase all momenta of particles by the same multiplicative factors $p_{i}(t)=p_{i}(0) \exp \left(H_{i} t\right)$. With sufficiently high drag, Langevin dynamics would bring

\footnotetext{
4 Although the exact nature of matter in the near- $T_{c}$ region is not yet understood, let us mention that the original "mixed phase" description, originating from the notion of the first-order phase transition, cannot be true, as "supercooling" and bubble formation expected in this case are not observed experimentally. Lattice gauge theory suggests a smooth crossover-type transition, with a high but finite peak in the specific heat. Recently there has been renewed interest in this region, after the so-called "magnetic scenario" for it has been proposed [46, 47], describing it as a plasma containing a significant fraction of magnetic monopoles.
}

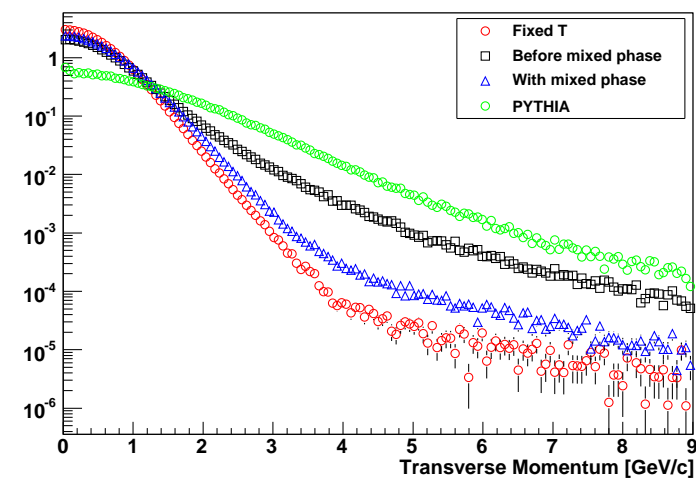

FIG. 11: (Color online.) The charm $p_{t}$-distribution after the mixed phase compared with the distribution without flow, the distribution orignating from PYTHIA, and the distribution before the mixed phase

the charm quarks rapidly to a thermal distribution, and since $M>>T$ it is a good approximation in this case to say that the heavy quarks have been "stopped". However, we will show below that at the "realistic" value used for the drag $\eta_{c}$ this does not happen during the time allocated to the mixed phase, there is instead ongoing "stopping" of the charm quarks relative to fluid elements. (This also will be important for the evolution of the azimuthal anisotropy $v_{2}\left(p_{t}\right)$ for single charm and for charmonium). The second scenario predicts $v_{2}\left(p_{t}\right)$ for single charm quarks which is far smaller than what is measured. We do not consider this scenario further even though something might be said for modelling charmonium in the mixed phase as interacting far less than single charm.

Several single charm $p_{t}$-distributions are shown in Figure 11 (normalized for simplicity to unity). The initial distribution after hard production predicted by PYTHIA is the largest at high $p_{t}$ : this is compared to the Langevin evolution before (squares) and after (triangles) the mixed phase, for a semi-central RHIC collision $(b=7 \mathrm{fm})$. In order to see that radial flow still is important, we have also shown what happens if Langevin evolution happen on top of unmoving fixed-T plasma (circles). This comparison demonstrates once again the main point of this paper, that for charm quarks and charmonium in a heavy-ion collision equilibration is never complete, even in momentum space: so the specific timescales of different phases of matter are of fundamental importance.

Unfortunately, in the near- $T_{c}$ region it is much less clear how to describe the $c-\bar{c}$ interaction. As we have learned from lattice data, the difference between freeenergy and potential-energy potentials are very drastic in this case: in the former case the tension (the force from the linear potential) disappears while in the latter it becomes about 5 times stronger than it is in vacuum. As discussed in refs 48, 49], the latter is presumably due to a metastable electric flux tube. 


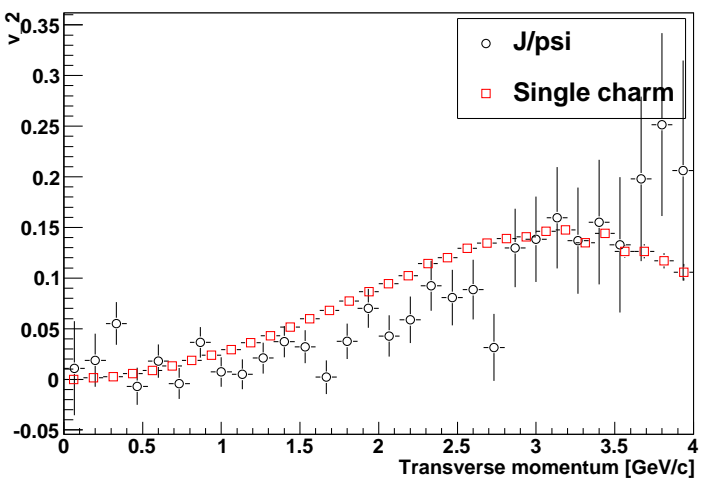

FIG. 12: (Color online.) The azimuthal anisotropy versus transverse momentum for both single charm and for $J / \psi$.

Which potential to use depends on timescales of the $c-\bar{c}$ dynamics, which are not well understood at this point. Therefore we took for now a conservative approach, assuming that at the near- $T_{c}$ stage charm pairs interact according to the simple Coulomb interaction $V=-\alpha_{s} / r$. Additionally, in our model for this phase we assume the interaction of the charm quarks with the medium can be modelled with the same Langevin dynamics with the temperature approximated as a fixed $T=T_{c}$ and the flow given as above. We found that with the simple Coulomb potential used in the mixed phase, the survival probability dropped slightly but not significantly: and although we do not discuss other possibilities in this work further, in principle this can be changed if the potential to be used has significant tension.

One final interesting observable would be a measurement of charmonium elliptic flow, characterized by the azimuthal anisotropy parameter $v_{2}=\langle\cos (2 \phi)\rangle$, induced by ellipticity of the flow on charmed quarks. A measurement with low statistics has been already made at PHENIX [50]: both PHENIX and STAR are working on higher statistics data on tape now. The result of our calculation of $v_{2}\left(p_{t}\right)$, both for single charm quarks and for the $J / \psi$, is shown in Figure 12 .

Greco, Ko, and Rapp also made predictions for the $v_{2}$ for $D$ and $J / \psi$ [51], based on a completely different scenario: in their case charm distributions are completely equilibrated and the charmonium states coalesce from them at hadronization. In spite of such difference, our predictions are similar: $v_{2}$ of $J / \psi$ should be less than the $v_{2}$ of single charm for low $p_{t}$, but then increase past the $v_{2}$ of single charm at $p_{t}>3 \mathrm{GeV}$. This shows, that the observation of charmonium $v_{2}$ can not be considered the argument for coalescence.

\section{SUMMARY}

We have studied a relaxation process of a $\bar{c} c$ pair produced by hard processes in heavy ion collision throughout the sQGP stage using hydrodynamics+Langevin. The main elements of the paper are: (i) inclusion of the interaction force between charm quarks and (ii) emphasis on deviations from equilibrium during the finite QGP lifetime.

Our main finding is that the lifetime of sQGP is not sufficient to reach the equilibrium distribution of the pairs in space, allowing for a significant fraction of $J / \psi$ $\sim 1 / 2$ to survive. This probability for charmonium dissociation in sQGP is larger than in earlier perturbative estimates, or for Langevin diffusion ignoring mutual interaction.

That is why there is no large difference between suppression at RHIC and SPS, in spite of the longer QGP lifetime in the former case. While the momentum relaxation is rather rapid, we found that later evolution reaches the so-called quasiequilibrium regime, which is maintained during all time of QGP expansion. The spatial distributions after some time develop a "core" in which $\bar{c} c$ pairs remain in close proximity due to the remaining effective attraction in sQGP, combined with a relatively slow leakage into a spreading tail toward large $r$. We propose quasiequilibrium as the clue to an explanation of the apparently thermal ratios of $\psi^{\prime}$ and $J / \psi$, especially at SPS.

The shape of the centrality dependence of our survival probabality is in agreement with data. Therefore, although we have not yet directly evaluated "nondiagonal" recombination, we think that most of the $J / \psi$ observed at SPS and RHIC are still from the "diagonal" pairs. This and other issues will of course be clarified, as more simulations and data in different kinematic domains become available.

The calculation is extended to the near- $T_{c}$ region known as a "mixed phase" in hydrodynamical calculations. Its duration for RHIC collisions is about $5 \mathrm{fm} / \mathrm{c}$, comparable to that of the QGP. We have used simple Hubble-like parameterization and minimal Coulomb potential, and predict both the charmonium $p_{t}$ spectra and azimuthal asymmetry parameter $v_{2}$ which is expected to be measured soon .

Acknowledgments. We thank P. Petreczky for providing lattice data on internal energy $V(T, r)$ used in this work. C. Young would like to thank K. Dusling for useful discussions on various topics in this work. We especially thank D. Teaney for permitting us use of his hydrodynamics+Langevin code and providing much needed assistance. This work is partially supported by the US-DOE grants DE-FG02-88ER40388 and DE-FG03-97ER4014.

\section{APPENDIX A: CLASSICAL VS. QUANTUM DYNAMICS}

In this paper, we take phase-space distributions of $\bar{c} c$ pairs and evolve them according to the Fokker- 
Planck/Langevin equations, which describe nonequilibrium evolution of phase-space distributions during the QGP era. After it is finished and the medium returns to the hadronic phase, our classical distribution $f(\vec{x}, \vec{p}, t)$ has to be projected into charmonium quantum states.

He we examine how classical and quantum dynamics correspond to each other in equilibrium, when both are easily available. We simplify by examining the thermal distributions for a Coulombic system, with $V \sim 1 / r$. One can calculate the density of states for the classical system:

$$
\begin{aligned}
Z & =\int d r d p(4 \pi)^{2} r^{2} p^{2} \exp \left(-\left(p^{2} / 2 \mu-e^{2} / r\right) / T\right) \\
& =\int d E \int d r d p(4 \pi)^{2} r^{2} p^{2} \exp (-E / T) \delta\left(E-p^{2} / 2 \mu+1 / r\right) \\
& =\int d E \exp (-E / T) \int_{\max (0, \sqrt{2 \mu E}}^{\infty} d p \frac{e^{2} p^{2}}{\left(p^{2} / 2 \mu-E\right)^{4}}
\end{aligned}
$$

As one can see, this integral is divergent for $E>0$. This means that this distribution is not normalizable and in thermal equilibrium all pairs are ionized. For $E<0$, we see from examining the partition function

$$
\rho(E) \propto(-E)^{-5 / 2}
$$

Now we calculate the quantum mechanical density of states for $E<0$,

$$
\rho(E)=\Sigma_{n=1}^{\infty} n^{2} \delta\left(E+\frac{C}{n^{2}}\right)
$$

which can be approximated by considering an integral:

$$
\begin{aligned}
\int_{-\infty}^{E} d E^{\prime} \rho\left(E^{\prime}\right) & =\Sigma_{i=1}^{\infty} i^{2} \theta\left(E+\frac{C}{i^{2}}\right) \\
& \sim E^{-3 / 2} \text { for large enough } \mathrm{E}
\end{aligned}
$$

Thus, $\rho(E) \sim E^{-5 / 2}$ for $E$ close to zero: therefore for highly excited states the correspondence principle holds and the classical thermal distribution is recovered. However, classical density of states is not so good of an approximation to the quantum-mechanical density of states for the lowest bound state.

We also tested whether Langevin simulations leads to the correct classical density of states, after some relaxation time. The result of evolving an ensemble of $\bar{c} c$ pairs at a fixed temperature according to classical Langevin dynamics shown in Fig 13 shows that equilibrium is indeed obtained.

\section{APPENDIX B: COALESCENCE PROBABILITY}

After all distributions at the end of the QGP era are determined, the next step is to calculate the probabilities of it materializing as a particular charmonium state.

One approach is based on the Wigner probability distribution. For any wavefunction $\psi(\mathbf{r})$,

$$
W_{\psi}(\mathbf{r}, \mathbf{p})=\frac{1}{\pi^{3}} \int d^{3} y \psi^{*}\left(\mathbf{r}+\frac{\mathbf{y}}{2}\right) \psi\left(\mathbf{r}-\frac{\mathbf{y}}{2}\right) e^{i \mathbf{p} \cdot \mathbf{y}}
$$

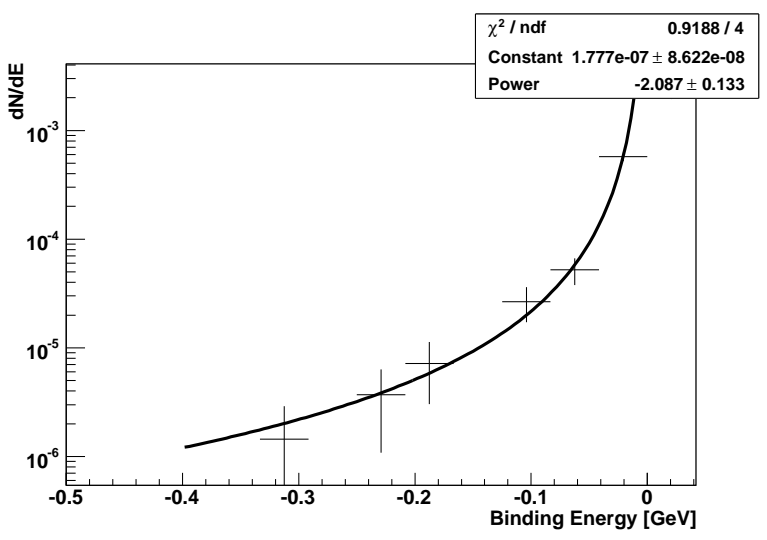

FIG. 13: The points show the density of states for an ensemble of $\bar{c} c$ pairs at a fixed temperature $T=1.5 T_{c}$ obtained by longtime Langevin evolution, compared to a fit with power 2.1 (curve), close to classical nonrelativistic thermal distribution.

The wave function for $\mathrm{J} / \psi$ was easily calculated numerically and then fitted to a Gaussian $\psi_{i}(x) \propto e^{-3.8 r^{2} / \mathrm{fm}^{2}}$. This leads immediately to the following parametrization of the $\mathrm{J} / \psi$ 's Wigner distribution's (here the constant is in $\mathrm{fm}^{-2}$ ) :

$$
W_{J / \psi}(x, p)=\frac{1}{\pi^{3}} \exp \left(-7.6 r^{2}-p^{2} / 7.6\right)
$$

Many properties for the Wigner transform so defined may be discussed but for our purposes here we should note that

$$
\int W_{\psi}(x, p) d p=|\psi(x)|^{2}
$$

and for another wavefunction $\chi(x)$ 's Wigner transform $W_{\chi}(x, p)$

$$
\int W_{\psi}(x, p) W_{\chi}(x, p) d x d y=\frac{1}{(2 \pi)^{3}}|\langle\psi \mid \chi\rangle|^{2},
$$

because this is what we need to properly normalize our distributions and to compute overlaps.

So, for our phase-space distribution at any given time $f(\vec{x}, \vec{p}, \tau)$, we model our probability of pair being measured in the $J / \psi$-state as

$$
P_{J / \psi}(\tau)=(2 \pi)^{3} \int f(\mathbf{r}, \mathbf{p}, \tau) W_{J / \psi}(\mathbf{r}, \mathbf{p}) d^{3} x d^{3} p
$$

We project the pairs onto the $J / \psi$-state not only at the onset of the calculation, but also throughout the time evolution, monitoring in this way the probability of $J / \psi$ production. We use this approach to estimate the coalescence probability for the distribution in Section III.

Later on we switched to the "energy projection method", which is ultimately used for projection into $\chi, \psi^{\prime}$ states as well as into $J / \psi$. It is based on the distribution over $\bar{c} c$ energy, in the pair rest frame, calculated 
with the (zero temperature) Cornell potential. One projection, used in Fig IIID, is to all bound states, defined by

$$
E_{C M}=V_{\text {Cornell }}(r)+\frac{p_{r e l}^{2}}{M_{c}}<0.88 \mathrm{GeV} .
$$

Later on, we differentiate between various charmonium states again by examining the $\bar{c} c$ pair's energy in its rest frame, and comparing it with the energies of solutions to Schrödinger's equation using the Cornell potential. For example, the lowest s-state solution, using the charm quark mass, has energy $E=0.33 \mathrm{GeV}$, therefore we count all $\bar{c} c$ pairs in our simulation with energy below $0.33 \mathrm{GeV}$ as existing in the $J / \psi$ state.
[1] E. V. Shuryak, Phys. Lett. B78 (1978) 150, Yadernaya Fizika 28 (1978) 796, Phys.Rep. 61 (1980) 71.

[2] M. E. Peskin, Nucl. Phys. B 156, 365 (1979). G. Bhanot and M. E. Peskin, Nucl. Phys. B 156, 391 (1979).

[3] D.Kharzeev and H.Satz, Phys.Lett.B334:155-162,1994, hep-ph/9405414

[4] A. Andronic, P. Braun-Munzinger, K. Redlich and J. Stachel, Phys. Lett. B 571, 36 (2003) arXiv:nucl-th/0303036.

[5] T. Matsui and H. Satz, Phys. Lett. B 178, 416 (1986).

[6] L. Grandchamp and R. Rapp, Nucl. Phys. A 715, 545 (2003) arXiv:hep-ph/0209141.

[7] H. van Hees and R. Rapp, Phys. Rev. C 71, 034907 (2005) arXiv:nucl-th/0412015.

[8] F. Karsch, M. T. Mehr and H. Satz, Z. Phys. C 37, 617 (1988).

[9] S. Datta, F. Karsch, P. Petreczky and I. Wetzorke, hep-lat/0208012. M. Asakawa and T. Hatsuda, Nucl. Phys. A715 (2003) 863c; hep-lat/0308034.

[10] G. Aarts, C. Allton, M. B. Oktay, M. Peardon and J. I. Skullerud, Phys. Rev. D 76, 094513 (2007) arXiv:0705.2198 [hep-lat]].

[11] E. V. Shuryak and I. Zahed, Phys. Rev. D 70, 054507 (2004), hep-ph/0403127

[12] Cheuk-Yin Wong, Phys.Rev.C72:034906,2005. hep-ph/0408020. W.M. Alberico, A. Beraudo, A. De Pace, A. Molinari Phys.Rev.D72:114011,2005. hep-ph/0507084

[13] A. Mocsy, P. Petreczky and J. Casalderrey-Solana, arXiv:hep-ph/0609205

[14] A. Mocsy and P. Petreczky, PoS LAT2007, 216 (2007) arXiv:0710.5205 [hep-lat]].

[15] Q. J. Ejaz, T. Faulkner, H. Liu, K. Rajagopal and U. A. Wiedemann, arXiv:0712.0590 [hep-th].

[16] F. Karsch and R. Petronzio, Phys. Lett. B 193, 105 (1987).

[17] K. Dusling and C. Young, arXiv:0707.2068 [nucl-th].

[18] H.Sato and K.Yasaki, Phys.Lett.B98 (1981) 153 M.Gylassy et al, Nucl.Phys. A402 (1983) 569.

$[19]$

[20] R. L. Thews, arXiv:hep-ph/0609121. R. Rapp, Eur. Phys. J. C 43, 91 (2005) arXiv:hep-ph/0502208.

[21] F. Karsch, D. Kharzeev and H. Satz, hep-ph/0512239

[22] G.Parisi,Phys.Lett.B131:393-395,1983

[23] J. Berges and I.-O. Stamatescu, Phys.Rev.Lett.95:202003,2005, hep-lat/0508030

[24] . O. Kaczmarek and F. Zantow, PoS LAT2005, 192 (2006) arXiv:hep-lat/0510094.

[25] E.V.Shuryak, PRC 55 (1997) 961; nucl-th/9605011

[26] G. D. Moore and D. Teaney, "How much do heavy quarks thermalize in a heavy ion collision?,"Phys. Rev. C 71,
064904 (2005) arXiv:hep-ph/0412346.

[27] J. M. Maldacena, Adv. Theor. Math. Phys. 2, 231 (1998) [Int. J. Theor. Phys. 38, 1113 (1999)] arXiv:hep-th/9711200.

[28] J. Casalderrey-Solana and D. Teaney, hep-ph/0605199

[29] C. P. Herzog, A. Karch, P. Kovtun, C. Kozcaz, and L. G. Yaffe, hep-th/0605158. S. S. Gubser, hep-th/0605182 A. Buchel, hep-th/0605178.S.-J. Sin and I. Zahed, hep-ph/0606049.

[30] B. A. Gelman, E. V. Shuryak and I. Zahed, Phys.Rev.C74:044909,2006, arXiv:nucl-th/0601029, Phys.Rev.C74:044908,2006, nucl-th/0605046.

[31] . J. Liao and E. Shuryak, arXiv:hep-ph/0611131

[32] E.M.Lifshitz and L.P.Pitaevskii, Physical Kinetics, Pergamon library of science, 1981.

[33] T. Sjöstrand, S. Mrenna and P. Skands, JHEP 0605, 026 (2006) arXiv:hep-ph/0603175.

[34] B. B. Back et al., Nucl. Phys. A 757, 28 (2005) arXiv:nucl-ex/0410022.

[35] I. Arsene et al. [BRAHMS Collaboration], Nucl. Phys. A 757, 1 (2005) arXiv:nucl-ex/0410020.

[36] S. S. Adler et al. [PHENIX Collaboration], Phys. Rev. Lett. 96, 012304 (2006) arXiv:nucl-ex/0507032.

[37] X. Dong, AIP Conf. Proc. 828, 24 (2006) [Nucl. Phys. A 774, 343 (2006)] arXiv:nucl-ex/0509038.

[38] O. Kaczmarek, S. Ejiri, F. Karsch, E. Laermann and F. Zantow, Prog. Theor. Phys. Suppl. 153, 287 (2004) arXiv:hep-lat/0312015.

[39] F. Karsch, D. Kharzeev and H. Satz, Phys. Lett. B 637, 75 (2006) arXiv:hep-ph/0512239.

[40] D. Kharzeev and M. Nardi, Phys. Lett. B 507, 121 (2001) arXiv:nucl-th/0012025.

[41] A. Adare et al. [PHENIX Collaboration], Phys. Rev. Lett. 98, 232301 (2007) arXiv:nucl-ex/0611020.

[42] H. Sorge, E. V. Shuryak and I. Zahed, Phys. Rev. Lett. 79, 2775 (1997) arXiv:hep-ph/9705329.

[43] S. Digal, P. Petreczky and H. Satz, Phys. Rev. D 64, 094015 (2001) arXiv:hep-ph/0106017.

[44] A. Adare et al. [PHENIX Collaboration], arXiv:0802.0050 [hep-ex].

[45] D. Teaney, J. Lauret and E. V. Shuryak, arXiv:nucl-th/0110037

[46] J. Liao and E. Shuryak, Phys. Rev. C 75, 054907 (2007) arXiv:hep-ph/0611131.

[47] M. N. Chernodub and V. I. Zakharov, Phys. Rev. Lett. 98, 082002 (2007) arXiv:hep-ph/0611228.

[48] J. Liao and E. Shuryak, Phys. Rev. C 77, 064905 (2008) arXiv:0706.4465 [hep-ph]].

[49] J. Liao and E. Shuryak, arXiv:0804.4890 [hep-ph].

[50] C. Silvestre and f. t. P. Collaboration, arXiv:0808.2925 [nucl-ex]. 
[51] V. Greco, C. M. Ko and R. Rapp, Phys. Lett. B 595, 202 (2004) arXiv:nucl-th/0312100. 